\title{
Analysis and Implementation of Enhanced Image Inpainting method using adjustable patch sizes
}

\author{
K Revathi ${ }^{1}$, B Janardhana Rao ${ }^{2}$ \\ ${ }^{1}$ Asst. Professor, CVR College of Engineering, Hyderabad, India, revathi.kandula@gmail.com \\ ${ }^{2}$ Assoc. Professor, CVR College of Engineering, Hyderabad, India, janardhan.bitra@gmail.com
}

\begin{abstract}
Image inpainting is the process of reconstruction of the damaged image and removal of unwanted objects in an image. In the image inpainting process patch priority and selection of best patch plays a major role. The patch size is also considered for producing good results in the image inpainting. In this paper patch priority is obtained by introducing a regularization factor $(\bowtie)$. The best patch selection is acquired by using the Sum of Absolute Difference (SAD) distance method. The results of inpainting are investigated with adjustable patch sizes of $5 \times 5,7 \times 7,9 \times 9$, $11 \times 11$, and $13 \times 13$ for the proposed method. The performance of these adjustable patch sizes is observed by using Peak Signal to Noise Ratio (PSNR) and Mean Square Error (MSE). The best suitable patch size for good inpainting is announced based on the values of PSNR and MSE.
\end{abstract}

Keywords: Inpainting, MSE, Patch size, PSNR, Regularization factor.

\section{INTRODUCTION}

The unwanted object removal in an image and reconstruction of the damaged image is generally termed as image inpainting. Image and video inpainting become the best research topic nowadays [1-5]. The basic image inpainting process is taken by using Partial Differential Equations (PDE) the process is called the diffusion method [6]. Later Total Variation (TV) model for inpainting is proposed by Chan and Shen using the anisotropic diffusion method [7]. These methods are well suited for structured images. It is modified further by curvature-driven diffusion [8]. In recent years enhanced methods in diffusion-based inpainting are implemented with fractional order variation models [9-10]. The diffusion-based inpainting methods give good results for images with a small filling region and fail for a large filling region. Texture synthesis and structure synthesis methods are used further to inpaint the images with textured and large filling regions [11-12]. This process of inpainting is called exemplar-based inpainting.

The exemplar-based inpainting was first proposed by Criminisi et al. [13]. Criminisi's method handles both structure and texture information of the images efficiently to inpaint the unknown region. In the exemplar-based inpainting method, the image is having an unknown region and a source region.
The best exemplars from the source region are used for filling the unknown region. The known region is called the source region and the unknown region is called target region. Criminisi's method was extended to a group of images instead of one image proposed by [14], which gives good results but more time-consuming. There are three major steps in the exemplar-based image inpainting process proposed by Criminisi et al., first step is to find the priority of the patches on the boundary of the target region. Next, determining the best matching patch from source region. Finally, the highest prioritized patch on the boundary of the target region is filed with the best matching patch from the source region and updating the boundary of the target region.

The Exemplar-based inpainting mainly depends on the patches created on boundary of the target region and patches in source region. The size of the patches affects a lot in the inpainting results in the exemplar-based method. This mainly concentrated on size of the patches taken in source and target region. The exemplar-based inpainting suffers from dropping effect due to sudden decrement of confidence term which leads priority drops to lower values for a smaller number of iterations.

In this paper proposed an alternate image inpainting method to avoid the dropping effect. The dropping effect is overcome by introducing regularization factor $(\epsilon)$ in the confidence term. The new inpainting method is implemented for different patch sizes of $5 \times 5,7 \times 7,9 \times 9,11 \times 11$, and $13 \times 13$. The inpainting results obtained from different patch sizes are comparing and identifying the suitable patch size for best inpainting results.

The rest of the paper is organized as follows: the enhanced image inpainting method using regularization for the priority of patch and selection of suitable patch size in section 2. The experimental results on different images with various patch sizes are reported in section 3. Section 4 concludes the work.

\section{ENHANCED IMAGE INPAINTING METHOD}

Let us consider an image $I$ shown in Fig. 1(a), $\Phi$ indicates the source region, $\Omega$ is the target region, and $\partial \Omega$ is the boundary of the target region. The patches with different sizes taken on boundary of the target region is represented with $\psi_{P}$, and $P$ is the center pixel of the patch. The patches taken on the boundary of the target region contain pixels of 
both source and target region. The complete process of exemplar-based image inpainting is shown in Figure 1.

\subsection{Enhanced Priority Function}

The priority of the patches on boundary of the target region is computed by,

$P(p)=G_{A}(P) * B(P)$

Here, $B(P)$ is the data term and $G_{A}(P)$ is the regularized confidence term given by

$G_{A}(P)=(1-\epsilon) A(P)+\epsilon$

The regularization factor $(\epsilon)$ is introduced to overcome the dropping effect obtained due to the direct multiplication of confidence term $A(P)$ and data term $B(P)$ in the Criminisi's method. The regularization factor is generally taken as 0.5 and 0.7 for best results of inpainting [15]. The confidence term indicates the information of best suitable pixels near to pixel $P$. The confidence term $A(P)$ is computed with,

$A(P)=\frac{\sum_{t \in \psi_{P} \cap \Phi} C(t)}{\left|\psi_{P}\right|}$

Here, $\mathrm{t}$ indicates the points on the coordinates in both $\psi_{P}$ and $\Phi$. Considering the confidence term value as 1 for source region and 0 for target region. Where $\left|\psi_{P}\right|$ represents the number of points in patch taken on boundary of the target region. The data term $B(P)$ gives the strength of isophotes coming towards the boundary of the target region, which is calculated by using equation (4) as

$B(P)=\frac{\left|\nabla I_{P}^{\frac{1}{2}} \cdot \vec{n}_{P}\right|}{255}$

The isophotes are lines that interconnect similar pixel values. $\nabla I_{P}^{\perp}$ is an isophote vector, $\vec{n}_{P}$ is a unit vector drawn normal to the boundary of the target region at pixel $P .255$ is the normalization factor value.

\subsection{Best Patch Searching Method}

The highest priority patch on the boundary of the target region is filled with best matching patch taken from the source region. The best matching patch also called exemplar is identified by computing the distance between the highest priority patch and the number of patches on the source region. The best exemplar patch is taken as

$\Psi_{q^{\prime}}=\arg \min _{\psi_{q} \in \Phi} d_{S A D}\left(\psi_{P}, \psi_{q}\right)$

Here, $d_{S A D}$ is the Sum of Absolute Difference distance, which is calculated with,

$d_{S A D}\left(\psi_{P}, \psi_{q}\right)=\sum\left|\psi_{P}-\psi_{q}\right|$

After finding the best exemplar patch, the exemplar patch is copied and pasted in the place of the highest prioritized patch on the boundary of the target region. Again, the same process repeats for the updated boundary of the target region till the completion of the entire target region.

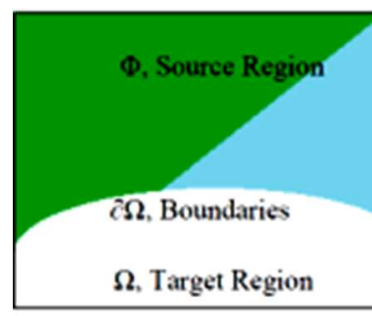

(a)

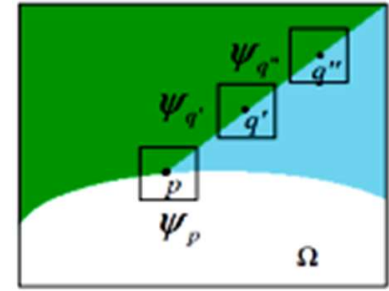

(c)

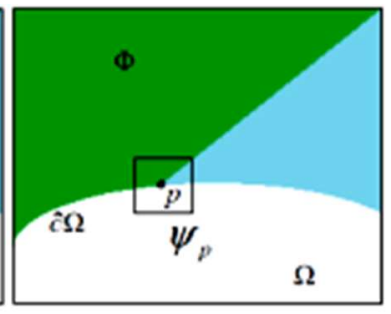

(b)

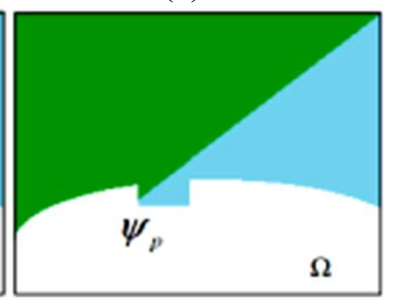

(d)
Figure 1: Exemplar-based Image Inpainting process [1]; (a) Image with source region and target region (b) target patch (c) target patch and source patches (d) filling result of one patch

\subsection{Adjustable Patch size}

The above process of finding the highest priority patch on the boundary of the target region and best exemplar patch searching is accomplished with fixed patch size. Here the entire process is doing with adjustable patch sizes of $5 \times 5$, $7 \times 7,9 \times 9,11 \times 11$, and $13 \times 13$. The whole inpainting process is completing with all patch sizes and investigates the best suitable patch size for good image inpainting results.

The complete process of enhanced image inpainting method with adjustable patch sizes is given in a step-by-step algorithm as

Algorithm:

\section{Read the Image I}

2. Form the source region $\Phi$ and target region $\Omega$

3. Draw the patches on the boundary $\partial \Omega$ with pixels on the boundary as the center point of the patch $\psi_{P}$

4. Find the highest priority patch on $\partial \Omega$ over $\psi_{P} i$, for $i=1,2,3, \ldots$.

5. Create patches on the source region as $\psi_{q}$ for $i=$ $1,2,3, \ldots$. find the best matching patch using SAD.

6. Copy and paste the best matching patch and update the boundary.

7. Repeat the whole process for different patch sizes.

\section{EXPERIMENTAL RESULTS}

The simulation is conducted in the system with a configuration of 4GB RAM, Intel Core i3 processor using MATLAB software. The experiment is carried out for different patch sizes of $5 \times 5,7 \times 7,9 \times 9,11 \times 11$, and $13 \times 13$ for images used in [2]. The performance metrics like Peak Signal to Noise Ratio (PSNR) and Mean Square Error (MSE) are computed for different images for analysis. The object removal in the input images and corresponding visual results 
K Revathi et al., International Journal of Emerging Trends in Engineering Research, 9(3), March 2021, 310 - 317

are shown in Figure 2 and Figure 3. Figure 2 shows the input images with an object to be removed and inpainted results for adjustable patch sizes of $5 \times 5,7 \times 7,9 \times 9,11 \times 11$, and $13 \times 13$ with regularization factor $\epsilon=0.5$. From the inpainted results of $\epsilon=0.5$, for different patch sizes, one can observe that results from the patch size $7 \times 7$ produce visually plausible inpainted images. The results for the remaining patch sizes found artifacts. Figure 3 shows the inpainted results for
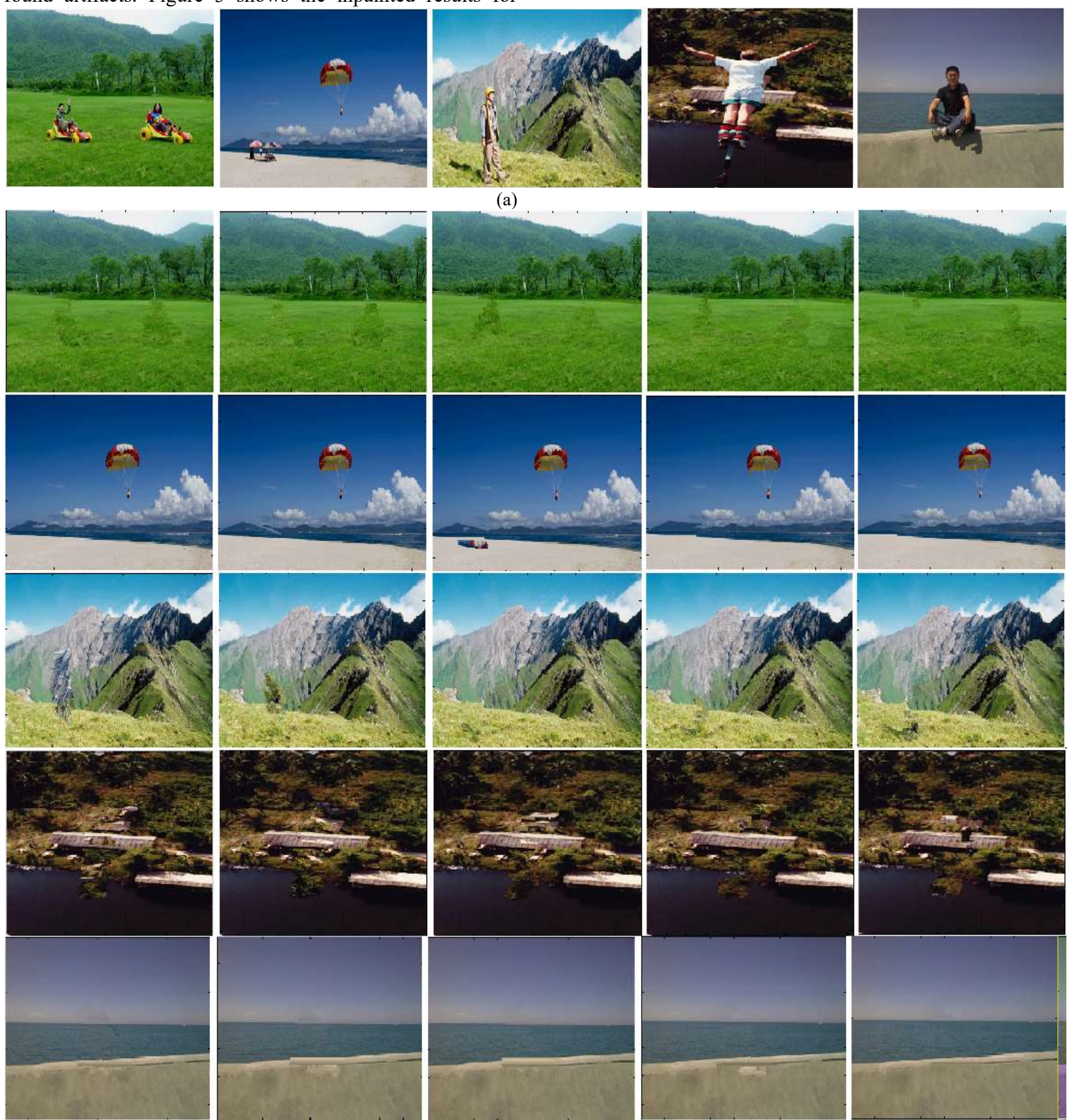

(a)

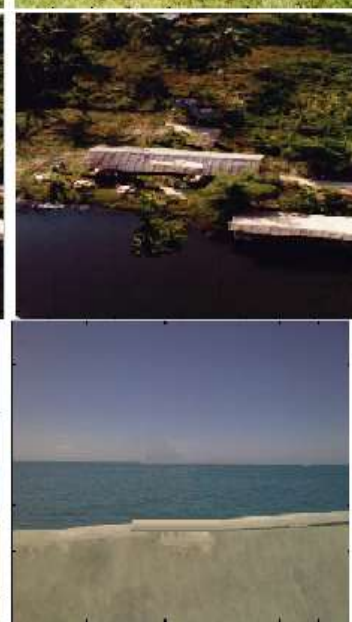

(b) (a)

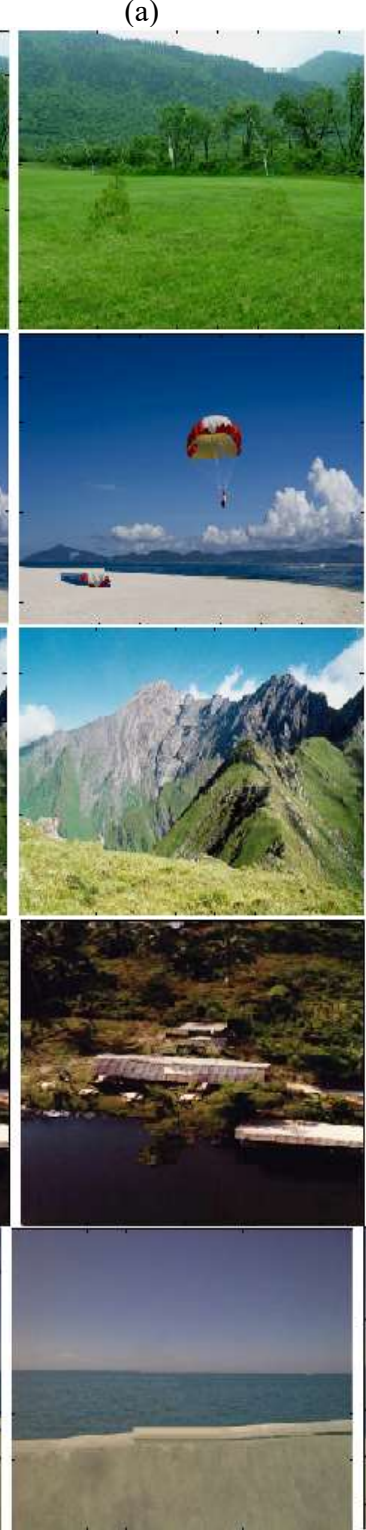

(c)

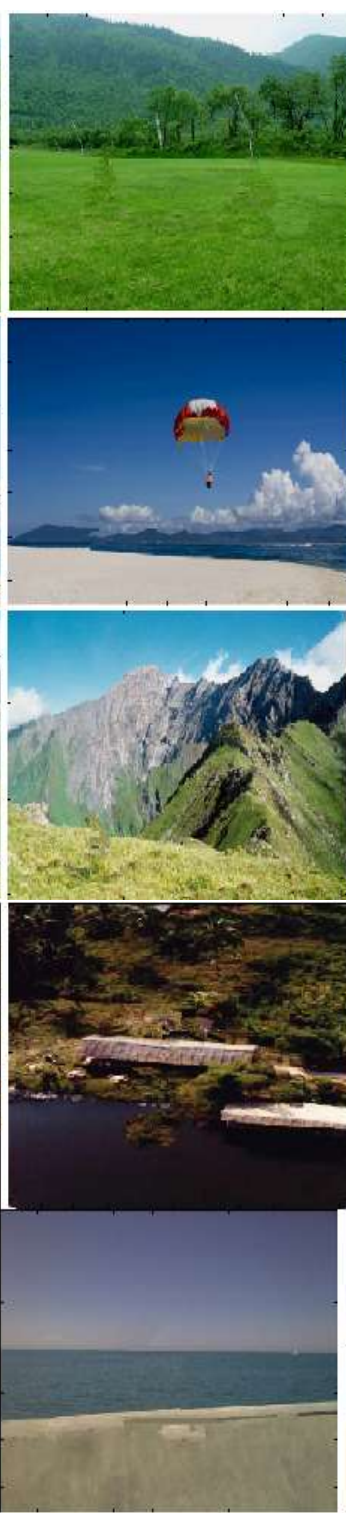

(d)

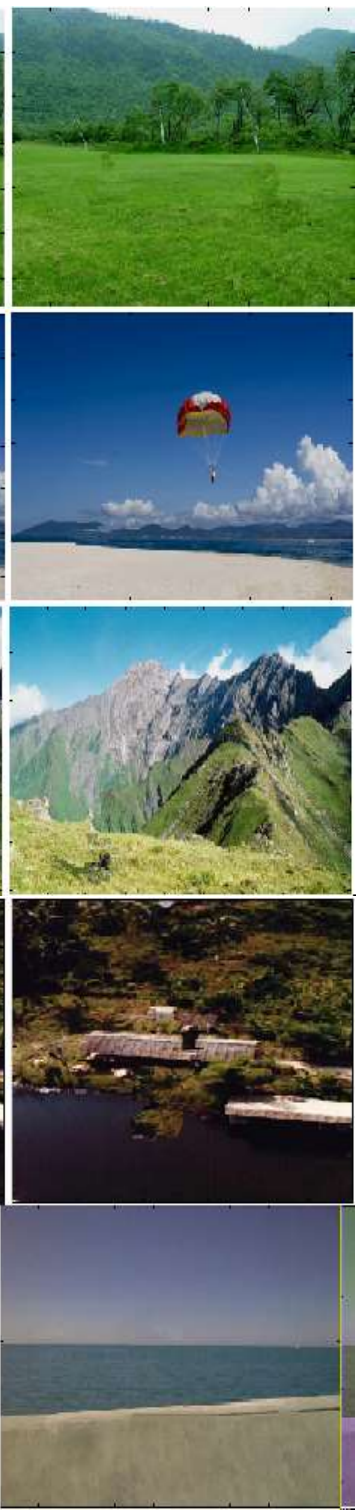

(e)

Figure 2: Object removal with for $\epsilon=0.7$; row (a), original Images; column (b), results for patch size $5 \times 5$; column (c), results for patch size $7 \times 7$; column (d), results for patch size $9 \times 9$; column (e), results for patch size $11 \times 11$; column (f), results for patch size $13 \times 13$ 

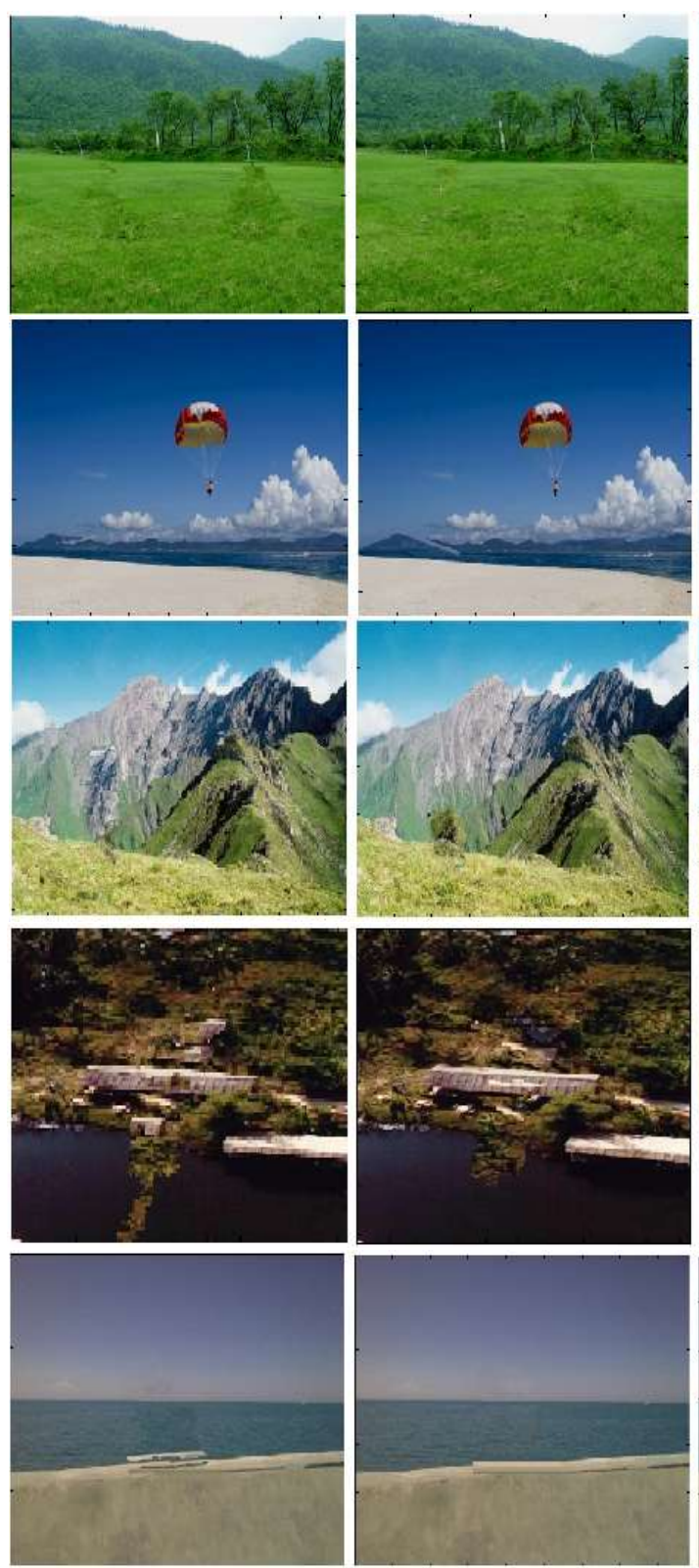

(a)

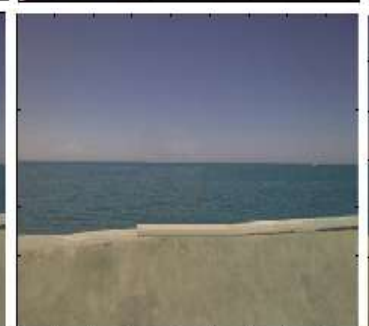

(b)
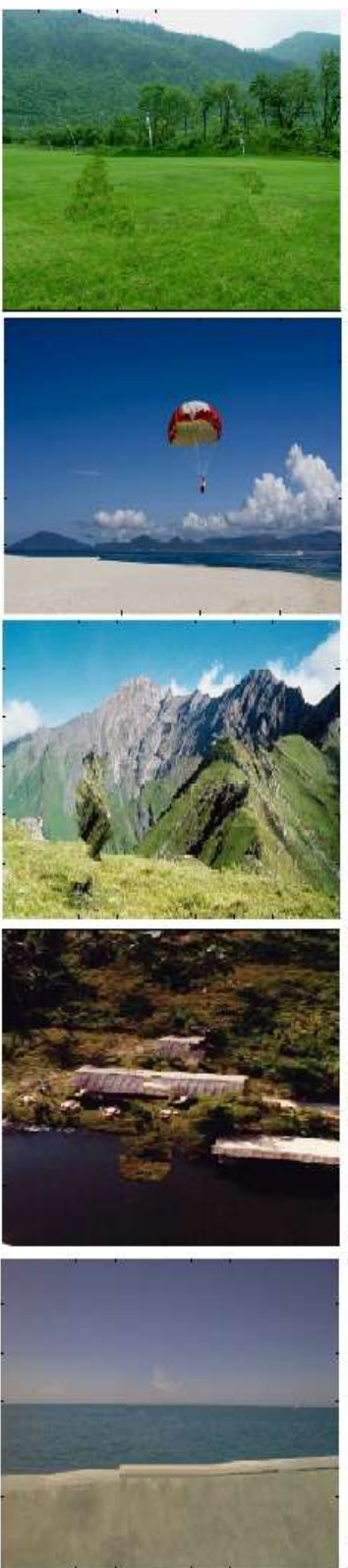

(c)
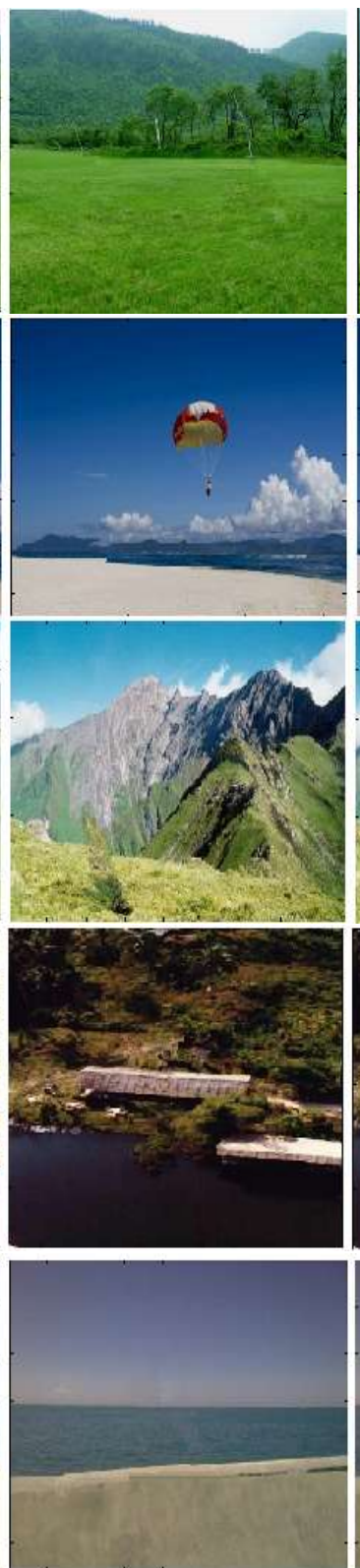

(d)
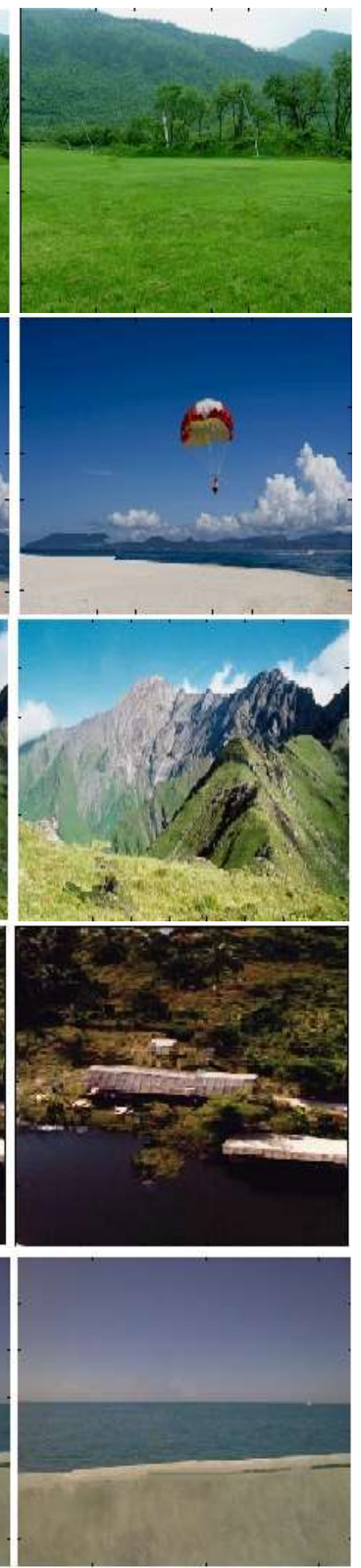

(e)

Figure 3: Object removal with for $\epsilon=0.7$; column (a), results for patch size $5 \times 5$; column (b), results for patch size $7 \times 7$; column (c), results for patch size $9 \times 9$; column (d), results for patch size $11 \times 11$; column (e), results for patch size $13 \times 13$

The quality metrics such as PSNR and MSE are calculated for all patch sizes and tabulated. The PSNR and MSE values for regularization factor $\epsilon=0.5$ are shown in Table I. From these values and a graphical representation, it is observed that the PSNR values are reasonably good for inpainted results obtained from patch size $7 \times 7$. The PSNR and MSE values for regularization factor $\epsilon=0.7$ of adjustable patch sizes are systemized in Table II. From these values, it is noticed that the PSNR value is better for results obtained using patch size $11 \times 11$. The time elapsed for the process of image inpainting is also computed. The time elapsed for $\epsilon=0.5$ and $\epsilon=0.7$ for adjustable patch sizes are reported in Table III. The time elapsed for image inpainting is more for the patch with less size $(5 \times 5)$ and very less for patch with the highest size $(13 \times 13)$. The time elapsed for the process is gradually reduced by increasing the patch size for inpainting. The time elapsed of image inpainting is inversely proportional to the size of the patch is used for inpainting. 
K Revathi et al., International Journal of Emerging Trends in Engineering Research, 9(3), March 2021, 310 - 317

Table I: PSNR (dB) and MSE Values for different patch sizes with Regularization factor $\epsilon=0.5$

\begin{tabular}{|c|c|c|c|c|c|c|c|c|c|c|}
\hline \multirow{2}{*}{$\begin{array}{l}\text { Input } \\
\text { Image }\end{array}$} & \multicolumn{2}{|c|}{$5 \times 5$} & \multicolumn{2}{|c|}{$7 \times 7$} & \multicolumn{2}{|c|}{$9 \times 9$} & \multicolumn{2}{|c|}{$11 \times 11$} & \multicolumn{2}{|c|}{$13 \times 13$} \\
\hline & MSE & PSNR & MSE & PSNR & MSE & PSNR & MSE & PSNR & MSE & PSNR \\
\hline Image 1 & 290.98 & 23.61 & 281.96 & 23.81 & 293.81 & 23.57 & 283 & 23.75 & 289.96 & 23.66 \\
\hline Image 2 & 101.59 & 28.26 & 100.91 & 28.92 & 143.68 & 26.76 & 120.05 & 27.53 & 109.34 & 27.91 \\
\hline Image 3 & 324.21 & 23.1 & 253.61 & 24.26 & 258.34 & 24.04 & 268.46 & 23.88 & 281.09 & 23.69 \\
\hline Image 4 & 1182.48 & 17.45 & 1148.78 & 17.54 & 1278.97 & 17.1 & 1255.18 & 17.2 & 1319.87 & 16.97 \\
\hline Image 5 & 438.69 & 21.75 & 416.92 & 22.39 & 461.61 & 21.53 & 473.14 & 21.42 & 439.58 & 21.74 \\
\hline
\end{tabular}

Table II: PSNR (dB) and MSE Values for different patch sizes with Regularization factor $\epsilon=0.7$

\begin{tabular}{|c|c|c|c|c|c|c|c|c|c|c|}
\hline \multirow{2}{*}{$\begin{array}{l}\text { Input } \\
\text { Image }\end{array}$} & \multicolumn{2}{|c|}{$5 \times 5$} & \multicolumn{2}{|c|}{$7 \times 7$} & \multicolumn{2}{|c|}{$9 \times 9$} & \multicolumn{2}{|c|}{$11 \times 11$} & \multicolumn{2}{|c|}{$13 \times 13$} \\
\hline & MSE & PSNR & MSE & PSNR & MSE & PSNR & MSE & PSNR & MSE & PSNR \\
\hline Image 1 & 292.22 & 23.59 & 295.61 & 23.58 & 293.81 & 23.59 & 282.42 & 23.78 & 285.73 & 23.76 \\
\hline Image 2 & 101.95 & 28.2 & 103.94 & 28.12 & 124.24 & 27.39 & 100.72 & 28.41 & 124.75 & 27.36 \\
\hline Image 3 & 300.63 & 23.41 & 368.49 & 23.26 & 368.73 & 22.51 & 259.46 & 24.41 & 272.43 & 23.83 \\
\hline Image 4 & 1127.06 & 17.67 & 1237.69 & 17.24 & 1287.5 & 17.07 & 1091.91 & 18.07 & 1335.04 & 16.92 \\
\hline Image 5 & 440.79 & 21.73 & 456.08 & 21.58 & 465.17 & 21.5 & 418.63 & 22.75 & 434.34 & 21.79 \\
\hline
\end{tabular}

Table III: Time Elapsed (sec.) for different patch sizes

\begin{tabular}{|c|c|c|c|c|c|c|c|c|c|c|}
\hline \multirow{2}{*}{$\begin{array}{l}\text { Input } \\
\text { Image }\end{array}$} & \multicolumn{5}{|c|}{$\epsilon=0.5$} & \multicolumn{5}{|c|}{$\epsilon=0.7$} \\
\hline & $5 \times 5$ & $7 \times 7$ & $9 \times 9$ & $11 \times 11$ & $13 \times 13$ & $5 \times 5$ & $7 \times 7$ & $9 \times 9$ & $11 \times 11$ & $13 \times 13$ \\
\hline Image 1 & 614.54 & 586.24 & 508.99 & 498.02 & 490.13 & 609.83 & 496.79 & 458.46 & 442.34 & 409.55 \\
\hline Image 2 & 173.45 & 132.30 & 128.65 & 124.78 & 124.77 & 138.08 & 135.10 & 128.76 & 127.45 & 126.26 \\
\hline Image 3 & 456.38 & 418.40 & 387.56 & 347.33 & 330.68 & 474.79 & 368.49 & 341.48 & 329.78 & 311.64 \\
\hline Image 4 & 211.59 & 161.48 & 140.76 & 136.27 & 130.11 & 190.17 & 149.56 & 129.18 & 127.89 & 120.94 \\
\hline Image 5 & 790.08 & 628.97 & 532.59 & 482.89 & 468.75 & 625.37 & 535.59 & 496.40 & 440.84 & 386.27 \\
\hline
\end{tabular}

The graphical comparative analysis of PSNR and MSE values for regularization factor $\epsilon=0.5$ are shown in Figure 4 and Figure 5 respectively. The comparative study of the proposed image inpainting method for adjustable patch sizes is evaluated by implementing with 5 different images. The patch size of $7 \times 7$ becomes the best patch size for producing good image inpainting results with an average PSNR 23.384 computed for 5 images. Which is the highest average PSNR value compared to other patch sizes. 


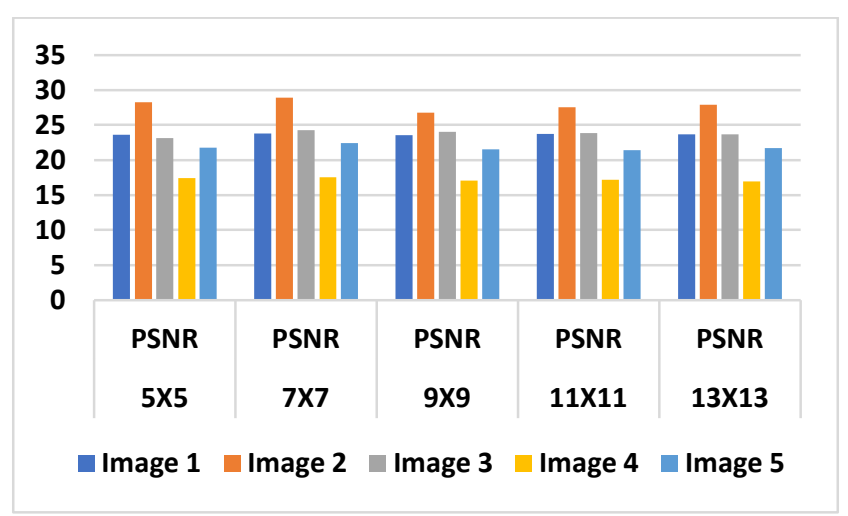

Figure 4. Comparison of PSNR values for $\boldsymbol{\epsilon}=\mathbf{0 . 5}$

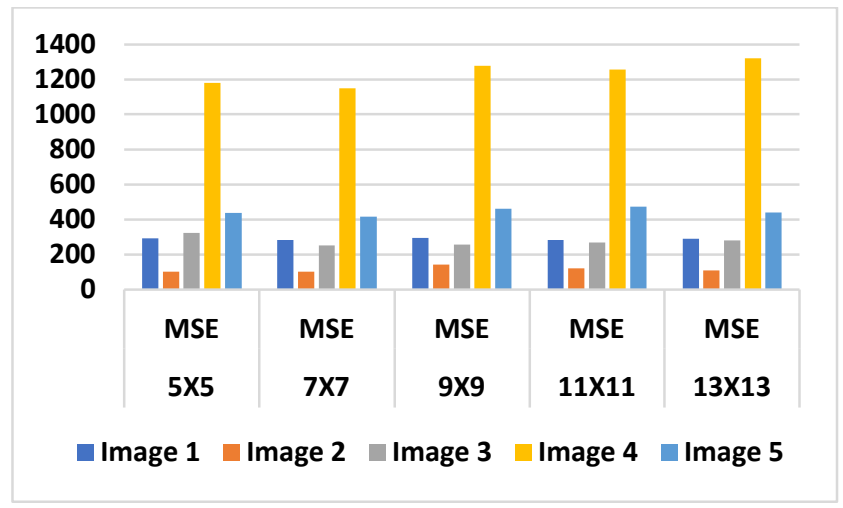

Figure 5. Comparison of MSE values for $\boldsymbol{\epsilon}=\mathbf{0 . 5}$

The pictorial representation of PSNR and MSE values for regularization factor $\epsilon=0.7$ are shown in Figure 6 and Figure 7 respectively. The average PSNR value for patch size $11 \times 11$ is 23.484 , which is best value compared to other patch sizes. So, from average PSNR values of the proposed inpainting method for different patch sizes and regularization factor, it is announced that $\epsilon=0.7$ with patch size $11 \times 11$ produce good inpainting results.

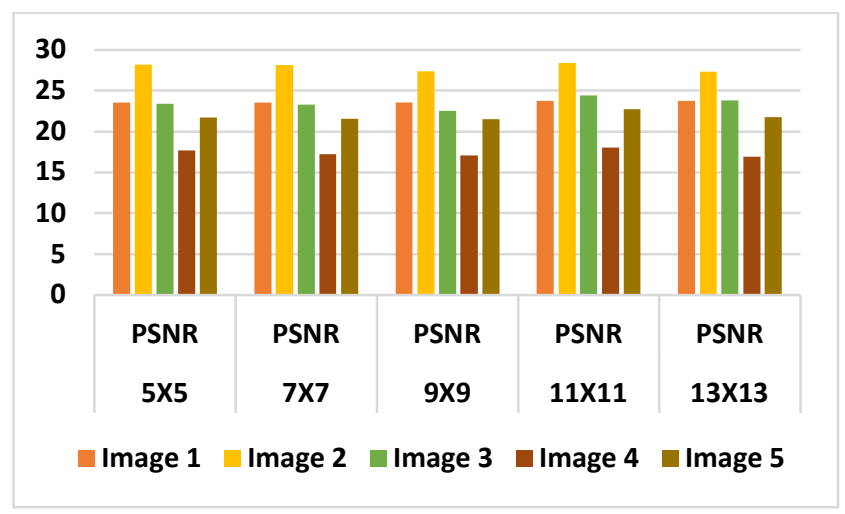

Figure 6. Comparison of PSNR values for $\boldsymbol{\epsilon}=\mathbf{0 . 7}$

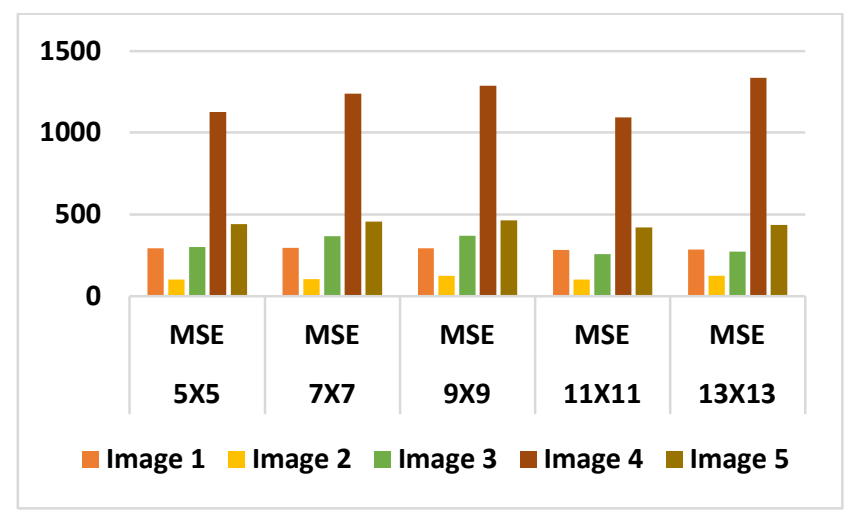

Figure 7. Comparison of MSE values for $\boldsymbol{\epsilon}=\mathbf{0 . 7}$

The graphical presentation of time elapsed for image inpainting process from proposed method for $\epsilon=0.5$ and $\epsilon=0.7$ are shown in Figure 8 and Figure 9 respectively. There is a tradeoff between PSNR, and time elapsed for the inpainting. If patch size is more it takes less time to inpaint the target region but with less PSNR. In the same way for less patch size, it takes much time to inpaint the target region. From this also we can say that $\epsilon=0.7$ and patch size $11 \mathrm{X} 11$ is the best choice for inpainting process.

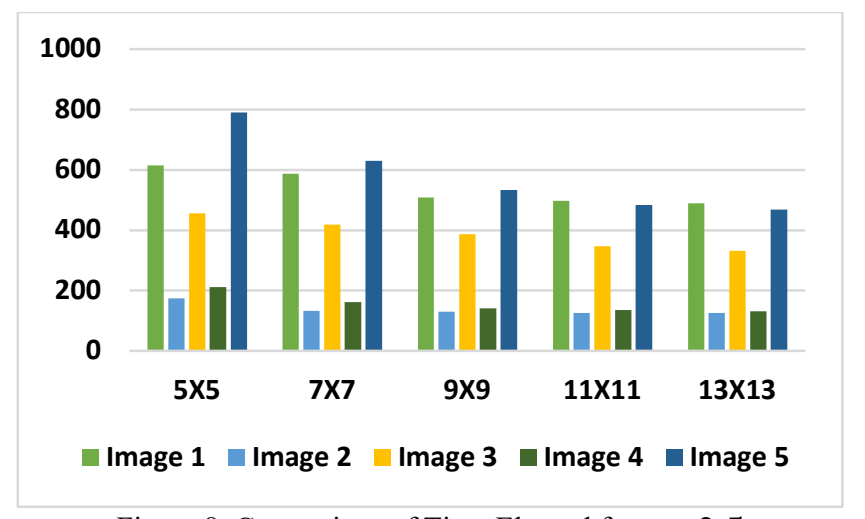

Figure 8. Comparison of Time Elapsed for $\boldsymbol{\epsilon}=\mathbf{0 . 5}$

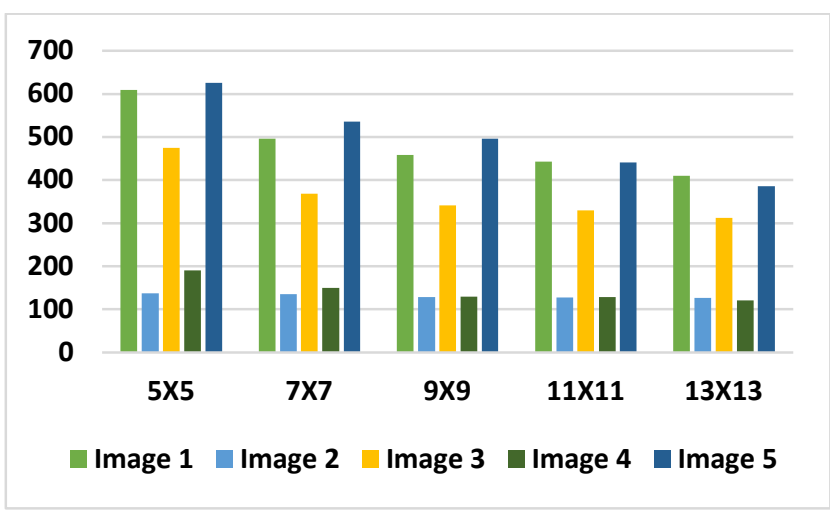

Figure 9. Comparison of Time Elapsed for $\boldsymbol{\epsilon}=\mathbf{0 . 7}$ 


\subsection{Comparison with Existing methods}

From the proposed work with two regularization factors for adjustable patch sizes with respective PSNR and MSE values, it is understanding that $\epsilon=0.7$ of patch size $11 \times 11$ producing good inpainting results. The inpainting results for $\epsilon=0.7$ of patch size $11 \times 11$, the proposed work is compared with state-of-art works in the literature [16][17][18][19][20] and [21]. The comparison of visual results for Lena and bungee image is shown in Figure 10 and Figure 11, respectively. The results from the proposed method are flawless and effective compared to existing methods. The comparison of PSNR values of different images with available methods in the literature is tabulated in Table III.

Table IV: Comparison with state-of-art works

\begin{tabular}{|c|c|c|c|c|}
\hline Method & Bungee & Lena & Baboon & Barbara \\
\hline Iizuka [16] & 18.46 & 36.44 & 33.43 & 38.3 \\
\hline Pathak [17] & 17.85 & 36.89 & 33.18 & 37.02 \\
\hline Yeh [18] & 18.27 & 37.2 & 33.02 & 38.85 \\
\hline Yu [19] & 18.33 & 37.15 & 33.25 & 38.55 \\
\hline Li [20] & 17.95 & 36.92 & 33.12 & 37.68 \\
\hline Chen[21] & 18.58 & 37.83 & 33.53 & 39.84 \\
\hline Proposed & 19.942 & 37.94 & $33 . .95$ & 39.97 \\
\hline
\end{tabular}

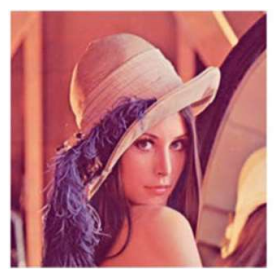

(a)

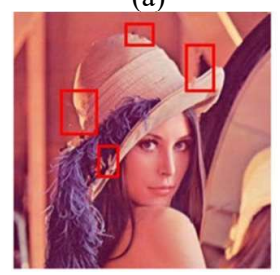

(d)

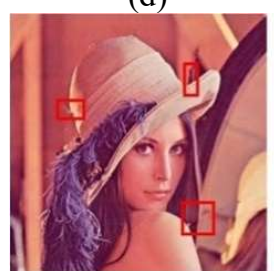

(g)

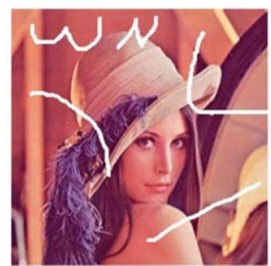

(b)

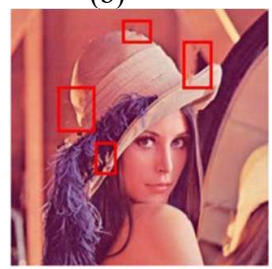

(e)

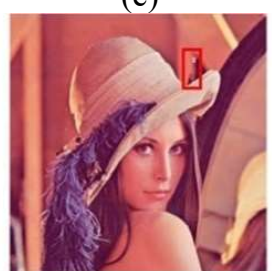

(h)

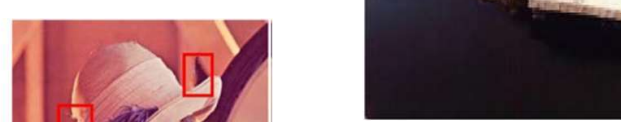

(g)

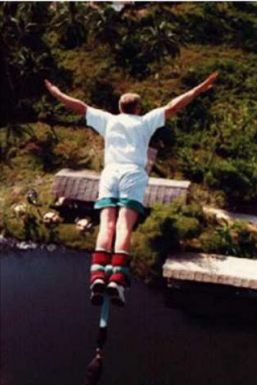

(a)

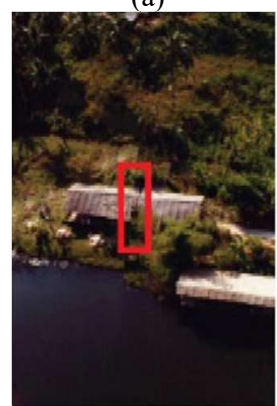

(d)

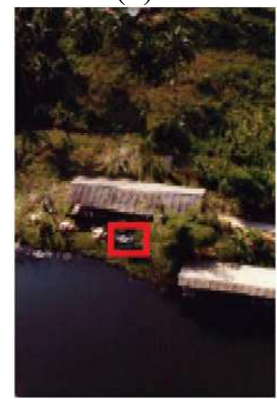

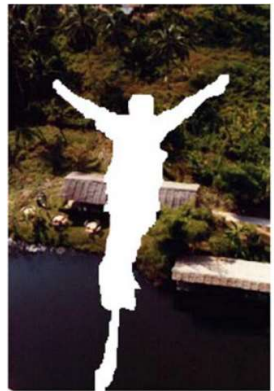

(b)

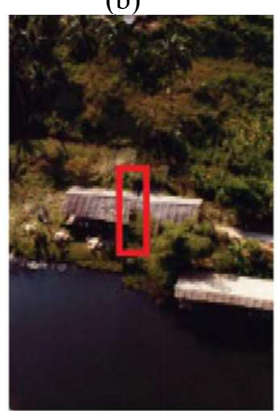

(e)

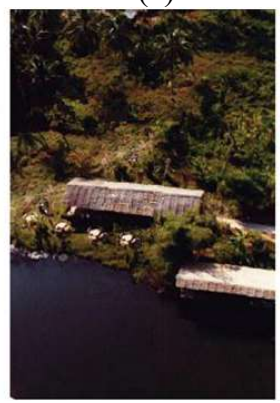

(h)

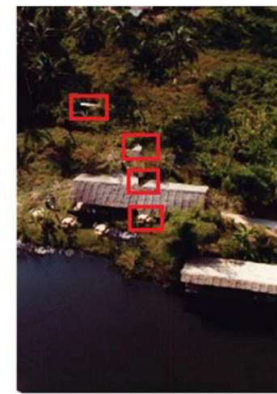

(c)

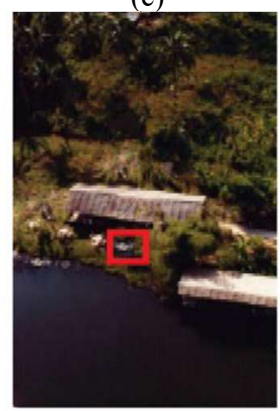

(f)

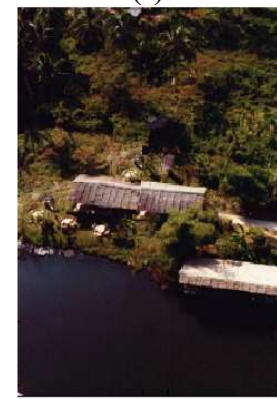

(i)
Figure11. Comparison with Existing Methods: Bungee (a) Original Image (b) Damaged Image (c) Result from Iizuka [16] (d) result from Pathak [17] (e) Result from Yeh [18] (f) Result from Yu [19] (g) Result from Li [20] (h) Result from Chen [21] (i) Result from Proposed method

\section{CONCLUSION}

The enhanced image inpainting method for the removal of objects in the image is presented. The method is carried out for two regularization factors $\epsilon=0.5$ and $\epsilon=0.7$ for adjustable patch sizes of $5 \times 5,7 \times 7,9 \times 9,11 \times 11$ and $13 \times 13$. According to the determined PSNR values, it is concluded that patch size $7 \times 7$ of $\epsilon=0.5$ and patch size $11 \times 11$ of $\epsilon=$ 0.7 produced good inpainting results. Considering the tradeoff between time elapsed for the inpainting method and PSNR values it is finally concluded that patch size $11 \times 11$ of $\epsilon=0.7$ turnout with best results for the image inpainting method.

\section{REFERENCES}

1. H. Wang et al., Exemplar-based image inpainting using structure consistent patch matching, Neurocomputing (2017), http://dx.doi.org/10.1016/j.neucom.2016.08.149. 
2. J. Wang, K. Lu, D. Pan, N He, B. Bao, Robust object removal with an exemplar-based image inpainting approach, Neurocomputing 123 (2014) 150-155.

3. D.Q. Chen, Y. Zhou, Inexact alternating direction method based on proximity projection operator for image inpainting in wavelet domain, Neurocomputing 189 (2016) 145-159.

4. Le, Thuc Trinh, et al. "Object removal from complex videos using a few annotations", Computational Visual Media 5.3 (2019): 267-291.

5. Xu, Rui, Xiaoxiao Li, Bolei Zhou, and Chen Change Loy. "Deep flow-guided video inpainting." In Proceedings of the IEEE Conference on Computer Vision and Pattern Recognition, pp. 3723-3732. 2019.

6. M. Bertalmio, G. Sapiro, V. Caselles, C. Bellester, Image inpainting, in: Proc. ACM SIGGRAPH, New Orleans, Lousiana, 2000, pp. 417-424.

7. T.F. Chan, J. Shen, Mathematical Models for local non-texture inpainting, SIAM J. Appl. Math. (2002) 1019-1043.

8. T.F. Chen, J. Shen, Non-Texture inpainting by Curvature-Driven Diffusion (CCD), J. Vis. Commun. Image Represent. 12 (2001) 436-449.

9. G. Sridevi, S.S. Kumar, "Image inpainting and enhancement using fractional order variational model", Defence Sci. J. 67(3), 308-315 (2017).

10. Sridevi, G., and S. Srinivas Kumar. "Image inpainting based on fractional-order nonlinear diffusion for image reconstruction", Circuits, Systems, and Signal Processing (2019): 1-16.

11. Efros, t. Leung, Texture synthesis by non-parametric sampling, in: Proc. ICCV, Kerkyra, Greece, 1999, pp. 1033-1038.

12. L. Wei, M. Levoy, Fast texture synthesis using tree-structured vector quantization, in: Proc. ACM SIGGRAPH, 2000, PP. 479-488.

13. Criminisi, P. Perez, K. Toyoma, Region filling and object removal by exemplar-based inpainting, IEEE Trans. Image Process. 13 (9) (2004) 1200-1212.

14. J. Hays, A. A. Efros, Scene completion using millions of photographs, Commun. ACM 51 (10) (2015) 87-94.

15. Janardhana Rao, B., Y. Chakrapani, and S. Srinivas Kumar. "Image Inpainting Method with Improved Patch Priority and Patch Selection." IETE Journal of Education 59.1 (2018): 26-34.

16. Iizuka, S., Simo-Serra, E., Ishikawa, H.: Globally and locallyconsistent image completion. ACM Trans. Graph. 36(4), 107:1-107:14 (2017).

17. Pathak, D., Krahenbuhl, P., Donahue, J., Darrell, T., Efros, A.: Context encoders: feature learning by inpainting. In: Proceedings of IEEE Conference on Computer Vision and Pattern Recognition, Las Vegas, NV, USA, 27-30 June 2016, pp. 2536-2544 (2016).

18. Yeh, A., Chen, C., Lim, T., Schwing, A., Hasegawa-Johnson, M.: Do M: Semantic image inpainting with deep generative models. In: Proceedings of IEEE Conference on Computer Vision and Pattern Recognition, Honolulu, HI, USA, 21-26 July 2017, pp.6882-6890 (2017).
19. Yu, J., Zhe, L., Yang, J., Shen, X., Xin, L., Huang, T.: Generative image inpainting with contextual attention. In: Proceedings of IEEE Conference on Computer Vision and Pattern Recognition, Salt Lake City, UT, USA, 18-22 June 2018, pp. 5505-5014 (2018).

20. Li, Y., Liu, S., Yang, J., Yang, M.: Generative face completion. In: Proceedings of IEEE Conference on Computer Vision and Pattern Recognition, Honolulu, HI, USA, 21-26 July 2017, pp. 5892-5900 (2017).

21. Chen, Yuantao, et al. "The improved image inpainting algorithm via encoder and similarity constraint." The Visual Computer (2020): 1-15. 